Thomas Hoffmann, Georgiana Baldea, Uwe Riedel, Thermodynamics and transport properties of metal/inert-gas mixtures used for arc welding, Proc. Combust. Inst. 32 (2), 3207-3214, 2009.

The original publication is available at www.elsevier.com

http://dx.doi.org/10.1016/j.proci.2008.06.195 


\title{
Thermodynamic and Transport Properties of
}

\section{Metal/Inert-Gas Mixtures used for Arc}

\section{Welding}

\author{
T. Hoffmann, G. Baldea, U. Riedel ${ }^{1}$ \\ University of Heidelberg, Interdisciplinary Center for Scientific Computing (IWR), \\ Im Neuenheimer Feld 368, 69121 Heidelberg, Germany
}

submitted to

$32^{\text {st }}$ Symposium (International) on Combustion

Montreal, Canada

August 3-8, 2008 


\begin{abstract}
The knowledge of thermodynamic and transport properties of gas mixtures at high temperature is important for many industrial applications. Spark ignition, re-entry problems of space-vehicles, gas dischargers, arc welding, and circuit breakers can be mentioned as examples. This paper focuses on metal/inert-gas mixtures in the temperature range from $300 \mathrm{~K}$ to $30000 \mathrm{~K}$, which are important for arc welding processes. As metals we consider iron, copper, aluminium and calcium and as inert gases nitrogen, argon and helium. The results are validated by comparison with transport properties from literature. However, this study also significantly extends and improves the transport model developed earlier [1,3]. The transport properties are calculated using Chapman-Enskog theory. The model is universally applicable and needs little input data. A multicomponent mixture formulation or simple mixing rules can be used.
\end{abstract}

Key words: new technology concepts, transport properties, thermodynamics, arc welding, metal vapor

$\overline{1}$ Corresponding author. Im Neuenheimer Feld 368, 69121 Heidelberg, Germany EMail: thomas.hoffmann@iwr.uni-heidelberg.de. Tel: +49 (6221) 54-8890. Fax: +49 (6221) 54-8884.

2 Research topic: new technology concepts, transport properties

3 Total length: approximately 5608 words (method 2 with $\mathrm{LAT}_{\mathrm{EX}}$ and elsart3p document class).

4 Approximate partial lengths: 3937 words for main text, 411 for references, 105 for Figs. 1-13 each (method 2 with $\mathrm{LT}_{\mathrm{E}} \mathrm{X}$ and elsart3p document class). 


\section{Introduction}

The prediction of transport properties is of general interest because CFD-type numerical simulations of many industrial applications need detailed information on transport processes including the electrical conductivity of the species present in the system. Spark ignition, re-entry problems of space-vehicles, gas dischargers, arc welding, and circuit breakers can be mentioned as examples. In this paper we focus on arc welding as an industrial application. However, this study also significantly extends and improves the transport model developed earlier [1]. In earlier publications, we presented a first version of the detailed transport model [1] and its application to high-temperature air (300 $\mathrm{K}<T<$ $30000 \mathrm{~K}$ ) at different pressures. Recent extensions included carbon dioxide and water at pressures of 0.1 mbar to 100 bar in the same temperature range [2] and the ion transport (and reactions) in lean methane flames [3].

The focus of this paper is to extend the model again to predict transport properties of various metal species. The motivation behind this is the important industrial application of arc welding and arc cutting. In this processes an electric arc is formed between an electrode and the workpiece protected by an inert gas (e.g. Ar, $\mathrm{He}, \mathrm{N}_{2}, \mathrm{CO}_{2}$ ). The most important workpiece materials are steel and aluminium but also copper, nickel, zirconium and titanium can be used. The knowledge of the transport properties enables the study of the behavior of the light arc. Here, an interesting aspect is the energy transport to the weldment which depends on the electrical and thermal conductivity. With our model we perform qualitative analysis which leads to a fundamental understanding of the metal vapors. In particular the influence of different metals and metal compositions on inert gases can be derived. We study the 
transport properties near the weldment only. The contamination of the inert gas with tungsten is neglected. On the basis of these calculations we obtain the input data needed to implement a two-dimensional model of the welding process in the near future.

\section{Composition}

Transport properties of different metal/inert-gas mixtures can be studied only if their compositions are known. A time-dependent rate equation model (HOMREA code [4]) allows the investigation of the dynamics of system considered and its relaxation into equilibrium. For an air mixture $\left(78.8 \% \mathrm{~N}_{2}, 21.2\right.$ $\% \mathrm{O}_{2}$ ) described through a 15 species model, our calculations at different temperatures (in the range $300-30000 \mathrm{~K}$ ) and 1 bar show that the main mixture species reach their equilibrium concentration not later than some $10-100 \mathrm{~ms}$. Since the time scale for arc welding processes is much larger, the assumption of local thermodynamic equilibrium is justified.

In order to determine the mixture compositions, homogeneous $0 \mathrm{D}$ equilibrium concentration simulations - based on Gibbs energy minimization including ideal gas behavior, mass conservation and electrical neutrality - have been performed using the DETCHEM package [5]. Considering different models similar to those from the literature, temperature and pressure dependent mole fraction profiles for different initial compositions have been obtained. Our metal/inert-gas models comprise mono- and diatomic neutral air and metal species, simply ionized metal anions as well as up to triply ionized cations. The following species are considered: $\mathrm{N}_{2}, \mathrm{~N}, \mathrm{~N}_{2}^{+}, \mathrm{N}^{+}, \mathrm{N}^{2+}, \mathrm{N}^{3+}, \mathrm{Ar}, \mathrm{Ar}^{+}, \mathrm{Ar}^{2+}$, $\mathrm{Ar}^{3+}, \mathrm{He}, \mathrm{He}^{+}, \mathrm{He}^{2+}, \mathrm{Fe}_{2}, \mathrm{Fe}, \mathrm{Fe}^{-}, \mathrm{Fe}^{+}, \mathrm{Fe}^{2+}, \mathrm{Fe}^{3+}, \mathrm{Cu}, \mathrm{Cu}_{2}, \mathrm{Cu}^{-}, \mathrm{Cu}^{+}, \mathrm{Cu}^{2+}$, 
$\mathrm{Cu}^{3+}, \mathrm{Al}_{2}, \mathrm{Al}, \mathrm{Al}^{-}, \mathrm{Al}^{+}, \mathrm{Al}^{2+}, \mathrm{Al}^{3+}, \mathrm{Ca}, \mathrm{Ca}^{+}, \mathrm{Ca}^{2+}, \mathrm{Ca}^{3+}$.

The thermodynamic data are extracted from the NASA database [6] and from Cheynet and Dubois [7]. A major challenge regarding the thermodynamic data is their extension for high temperatures and for higher ionized species. Equilibrium compositions for temperatures higher than the upper temperature limit given in the thermodynamic dataset have been calculated assuming constant heat capacity. Since the total Gibbs energy is derived from the enthalpy term and the negative entropie term, both terms being underestimated, partial error compensation justifies this approach. Neglecting the differences in entropy, the missing thermodynamic ion data for higher ionized species have been derived from the neutral or precursor ion by adding the corresponding ionization energy to its enthalpy.

\section{Model description}

The transport model is based on the theory of dilute gases of Chapman and Enskog $(\mathrm{CE})[8,9]$. The transport coefficients in multicomponent mixtures are determined from binary transport coefficients which are derived from collision integrals. This model uses precalculated and tabulated reduced collision integrals computed from different intermolecular potential functions.

\subsection{Interaction Potentials}

In a plasma different kinds of binary collisions occur, depending on the charge number of the collision partners. Therefore, various interaction potential functions are considered. 


\subsubsection{Neutral-Neutral Interaction}

Interactions between neutral species are described by the Stockmayer-(12-63)-potential [11] and the exponential repulsive potential. The Stockmayerpotential

$$
\varphi(r)=4 \epsilon_{i k} \cdot\left[\left(\sigma_{i k} / r\right)^{12}-\left(\sigma_{i k} / r\right)^{6}+\delta\left(\sigma_{i k} / r\right)^{3}\right]
$$

is used at reduced temperatures $T^{*}=k_{b} T / \epsilon_{i k}<10$, with the Boltzmann constant $k_{b} . \sigma_{i k}$ and $\epsilon_{i k}$ are derived from the Lennard-Jones parameters of the pure species $\mathrm{i}$ and $\mathrm{k}$ by special combination rules [8]. The parameter $\delta$ includes the variation from homopolar behavior.

For $T^{*}>10$ the following exponential repulsive potential [12] is used:

$$
\varphi(r)=V_{i k} e^{-r / \beta_{i k}}
$$

The potential parameters are calculated from the Born-Mayer parameters of the pure species [10].

\subsubsection{Neutral-Ion Interaction}

The (16-6-4)-potential [13] describes the interactions between neutrals and ions for $T^{*}<10$ :

$$
\begin{aligned}
\varphi(r)= & \frac{16 \hat{\epsilon}}{16(3+\gamma)-12(1+\gamma)} . \\
& \left((12 / 16)(1+\gamma)\left(r_{m} / r\right)^{16}-4 \gamma\left(r_{m} / r\right)^{6}-3(1-\gamma)\left(r_{m} / r\right)^{4}\right) .
\end{aligned}
$$

The depth of the potential well $\hat{\epsilon}$ and position $r_{m}$ are estimated from polarizabilities. The parameter

$$
\gamma=\frac{\left(2 / Z_{i}^{2}\right)\left(C_{i k} / e^{2}\right)+\alpha_{q, k}}{\alpha_{k} r_{m}^{2}}
$$


describes the relative strength between the dispersion and the polarization force. $Z_{i}$ is the charge number of ion $i, \alpha_{q, k}$ the quadrupole polarizability of the neutral $k$, and $C_{i k}$ the dispersion coefficient of the binary mixture. $e$ denotes the elementary charge. The binary dispersion coefficients are determined by the combination rule [14]

$$
C_{i k}=\frac{2 C_{i} C_{k}}{\left(\alpha_{k} / \alpha_{i}\right) C_{i}+\left(\alpha_{i} / \alpha_{k}\right) C_{k}}
$$

For $T^{*}>10$ the exponential repulsive potential mentioned above is used.

The collision integrals of electron-neutral interaction are calculated by numerical integration of the momentum collision cross sections or differential collision cross sections.

\subsubsection{Ion-Ion Interaction}

Interactions between charged particles are described by the screened Coulomb potential

$$
\varphi(r)=\frac{\left(Z_{i} e\right)\left(Z_{k} e\right)}{4 \pi \epsilon_{0} r} e^{-r / \lambda_{D}}
$$

with the charge numbers $Z_{i}$ and $Z_{k}$ and the temperature dependent Debyelength $\lambda_{D}[15,16]$.

\subsection{Resonant Charge Transfer}

The resonant charge transfer $\left(A+A^{+} \rightarrow A^{+}+A\right)[17]$ is a quantum-mechanical symmetry effect which decreases the binary diffusion coefficients. It is modeled by a simple approximation of collision cross sections:

$$
\left(Q_{\mathrm{rct}}(E)\right)^{1 / 2}=A_{\mathrm{rct}}-B_{\mathrm{rct}} \ln \left(E / k_{b}\right) .
$$


$E$ is the kinetic energy. This leads to the reduced collision integrals

$$
\Omega_{\mathrm{rct}}^{(1,1) *}=\frac{1}{\pi \sigma_{i k}^{2}}\left(\left[A_{\mathrm{rct}}-B_{\mathrm{rct}}(\Psi(3)+\ln T)\right]^{2}+\Psi^{\prime}(3) B_{\mathrm{rct}}^{2}\right)
$$

\subsection{Multicomponent Transport Formulation}

The ordinary diffusion coefficients of the mixture are determined by solving a linear equation system by matrix inversion. The thermal diffusion coefficients are calculated from binary thermal diffusion ratios. Ambipolar effects are included $[18,19]$. The viscosity and the frozen thermal conductivity of the mixture are calculated using conjugate gradient methods [20]. The EuckenHirschfelder formalism [21] takes contributions from internal energies into account. Therefore, the thermal conductivity of the pure species is calculated by considering a modified Eucken-correction [22]. Contributions due to chemical reactions are included as (ambipolar) diffusion of the enthalpy of the species. The transport properties are computed in the first approximation of the CE-theory, except for the transport properties of electrons. Simplified expressions for the calculation of the thermal conductivity (third approximation) and diffusion coefficients (second approximation) are used [23]. The electrical conductivity is derived from the ordinary diffusion coefficients.

\section{Results}

\subsection{Equilibrium Compositions}

The equilibrium composition simulations provide the necessary species composition which is input to the computation of the transport coefficients. But 
already the equilibrium composition provides a better understanding of the transport properties. In Figures 1-3, the mole fractions of the most important species (with mole fractions higher than 0.001) are plotted on a logarithmic scale against temperature (up to $30000 \mathrm{~K}$ ) for three different iron/inert-gas mixtures consisting of $10 \%$ iron and $90 \%$ nitrogen, argon, and helium at 1 bar.

Figure 1 demonstrates the characteristic metal behavior on the basis of the equilibrium concentrations in the Fe/Ar mixture. At room temperature, the iron dimer dominates, whereas above $1000 \mathrm{~K}$, due to its decomposition, the Fe atom concentration increases considerably. Further temperature enhancement favors the ionization of the single iron atoms to $\mathrm{Fe}^{+}$with concentrations larger than 1 mole- $\%$ in the temperature range of $6000-18000 \mathrm{~K}$. Above $1500 \mathrm{~K}$, the second ionization to $\mathrm{Fe}^{2+}$ becomes more and more important - at the expense of $\mathrm{Fe}^{+}$. Up to $30000 \mathrm{~K}$ even the triply ionized iron cations contribute to the mixture composition with about 2 mole-\%. For higher ionized species, simulations revealed negligible concentrations. Hence, for each inert gas or metal element up to triply ionized cations are considered. Anions might play in general a certain role [2]; however, for the present work, their contribution is insignificant.

With regard to the transport properties of the different metal/inert gas mixtures, not only the influence of the metal, but first and foremost - due to its large contribution of $90 \%$ - the inert gas behavior has to be analyzed. Figures 2 and 3 enable a comparison between the three different iron systems, illustrating - in analogy to Figure 1 showing iron as example for a metal species - the equilibrium concentrations as a function of temperature of the most important nitrogen, argon and helium species respectively. In all three cases, the 
metal : inert-gas ratio is $1: 9$.

At room temperature, the thermodynamically stable nitrogen molecule dominates the mixture composition. With increasing temperature, its dissociation into single $\mathrm{N}$ atoms is enhanced and dominates over its ionization to $\mathrm{N}_{2}^{+}$. Above $10000 \mathrm{~K}$ the first ionized species become noticeable. From $22000 \mathrm{~K}$ to $30000 \mathrm{~K}$ doubly ionized $\mathrm{N}^{2+}$ species arise; for $\mathrm{N}^{3+}$ the mole fraction never exceeds 0.001. In addition to the temperature profile of the nitrogen species, Figure 2 also shows the characteristics of the electron concentration profile. Despite of the low metal percentage in the mixture, but due to the low metal ionization energy (7.90 eV for Fe), below $10000 \mathrm{~K}$ electrons result mainly from the first ionization of Fe. For higher temperatures, the electron profile reflects the ionization of the atomic nitrogen.

Figure 3 shows for the argon and helium species similar concentration profiles as in case of nitrogen. Since the first and second ionization energies for $\mathrm{N}$ and Ar are in the same range $(14.52 \mathrm{eV}$ vs. $15.76 \mathrm{eV}$ for the first and $29.60 \mathrm{eV}$ vs. $27.63 \mathrm{eV}$ for the second ionization respectively), their species-temperature profiles differ only in the low temperature region (due to the high dissociation energy of the nitrogen molecule) considerably. Argon needs only $40.74 \mathrm{eV}$ for its third ionization compared to $47.45 \mathrm{eV}$ for nitrogen. Therefore the mole fractions for $\mathrm{Ar}^{3+}$ are larger than 0.001 around $30000 \mathrm{~K}$.

Since the first and second ionization energies $(24.59 \mathrm{eV}$ and $54.42 \mathrm{eV}$ respectively) of $\mathrm{He}$ are much higher than for $\mathrm{Ar}$ or $\mathrm{N}$, the He profile in Fig. 3 decreases much slower with temperature. $\mathrm{He}^{+}$occurs at much higher temperatures (above $17000 \mathrm{~K}$ ) in mole fractions larger than 0.01. Due to its very high second ionization energy of $54.42 \mathrm{eV}, \mathrm{He}^{2+}$ does not reach significant 
concentrations below $30000 \mathrm{~K}$.

Additional studies for other metals $(\mathrm{Cu}, \mathrm{Al}, \mathrm{Ca}, \mathrm{Ag})$ lead to similar observations. The differences in the metal species profiles in mixtures with different inert gases are less compared to the deviations between the inert gas species themselves. In general, the anionic metal species concentrations are negligible. The remarkable influence of the low ionizing metal species on the electron concentration suggests a similar significant influence on the transport properties.

\subsection{Transport properties}

\subsubsection{Iron/Inert Gas Mixtures}

The equilibrium compositions for iron and inert gases presented above already suggests a great influence of metal vapors on the transport properties. In Figure 4 the electrical conductivity of these compositions with $10 \%$ iron and $90 \%$ inert gas plotted against temperature is shown. The influence of ionization on the electrical conductivity is obvious. With the ionization of the gas, the electrical conductivity increases, because it strongly depends on the electron density. Pure helium has as expected the lowest electrical conductivity followed by argon and nitrogen which have similar values. The effects of the iron addition to argon and nitrogen are significant below $10000 \mathrm{~K}$ and for helium below $17000 \mathrm{~K}$. The second ionization of iron which occurs at about $17000 \mathrm{~K}$ has only little influence.

Figure 5 shows the thermal conductivity of iron/inert-gas mixtures with $10 \%$ iron. The thermal conductivity of pure helium below $15000 \mathrm{~K}$ is dominated by the internal and the translational contribution of the neutral particles. Over 
$15000 \mathrm{~K}$ the translational conductivity of the electrons and the contribution of chemical reactions of the ionization processes to the conductivity become significant. The addition of iron to helium results in lower internal and translational contribution of neutrals which leads to a lower total thermal conductivity. The difference between the Fe/Ar mixture and pure argon is based on the contribution of reaction. In the Fe/Ar mixture an ionization peak for iron at $8000 \mathrm{~K}$ is observed and the ionization peak of argon at $15000 \mathrm{~K}$ is lowered. The $\mathrm{Fe} / \mathrm{N}_{2}$ mixture differs from pure nitrogen between 10000 and $20000 \mathrm{~K}$. In this temperature range the contribution of reactions, which is based on the first ionization of nitrogen, is lowered because of the iron content. The peaks of pure nitrogen and the Fe/Ar mixture between $5000 \mathrm{~K}$ and $9000 \mathrm{~K}$ are almost equal. The explanation is that both the first ionization of iron and the dissociation of nitrogen occur in this temperature range and compensate each other.

Figure 6 shows the influence of iron contamination on the viscosity of the inert gas. For the Fe/ $\mathrm{N}_{2}$ mixture the effect of the metal vapor is small. In contrast, the viscosity for $\mathrm{Fe} / \mathrm{Ar}$ and especially for $\mathrm{Fe} / \mathrm{He}$ mixtures shows a notable impact. The viscosity is lowered above $5000 \mathrm{~K}$ and the increase of the viscosity is less pronounced. Besides that, the maximum of viscosity is shifted to higher temperatures. These effects are caused by the low ionization energy of iron. The relatively long-ranged Coulomb forces between charged particles decrease their mobility with increasing charged particle densities. This results in a reduction of viscosity. 


\subsubsection{Metal/Argon Mixtures}

The next focus of our studies is the influence of different types of metals on the transport properties. As inert gas argon is chosen. Figure 7 shows the electrical conductivity. As expected, the metals with the lowest ionization potential calcium and aluminium have the largest electrical conductivity below $10000 \mathrm{~K}$. Copper contamination has the lowest influence on the electrical conductivity. An interesting result can be seen for the argon mixture with 10 $\%$ iron and $1 \%$ calcium: the influence of the addition of as little as $1 \%$ calcium causes a significant increase of the electrical conductivity. The explanation for this result is that the released electrons from the ionization of the calcium dominates the mixture beneath $6000 \mathrm{~K}$. The lower ionization potential of calcium leads to a significant amount of electrons in this temperature even though the mole fraction is significant smaller than the mole fraction for the iron. This is an important result that shows that it will be important to consider the different components of a studied steel, especially if they have a low ionization potential.

In Fig. 8 the thermal conductivities of metal/argon mixtures are shown. The thermal conductivities of the different metals are quite similar. This is the case because of similar contributions from chemical reactions. They show maxima corresponding to the dissociation of metal dimers at about $2000 \mathrm{~K}$ and to the ionization energies of the metal at about $7000 \mathrm{~K}$. The first peak for calcium is absent because the calcium dimer is not considered. The largest influence on the thermal conductivity shows again calcium and the least effect has the addition of copper. 


\subsection{Validation}

The metal/inert-gas mixtures investigated most are $\mathrm{Cu} / \mathrm{N}_{2}[24-26]$ and $\mathrm{Cu} / \mathrm{Ar}$ mixtures [28-33]. These compositions do not really fit in the context of arc welding but they are well documented and are therefore used to demonstrate the predictive capabilities of our transport model. Besides these publications, mixtures with different metals (like calcium, aluminium, silver, copper, and iron) and with different inert gases (like nitrogen, argon, and helium) are also found in literature. However, the problem is that most of those results published are based on a single transport model. Therefore, to validate our model, we decided to choose the well studied $\mathrm{Fe} / \mathrm{Ar}, \mathrm{Cu} / \mathrm{Ar}$ and $\mathrm{Cu} / \mathrm{N}_{2}$ mixtures for which at least two or more results from different research groups are available.

\subsubsection{Copper/Nitrogen Mixture}

In Fig. 9 the electrical conductivity of a $\mathrm{Cu} / \mathrm{N}_{2}$ mixture with $10 \%$ copper is shown. Our results are in sufficient agreement with the results of other groups. Gleizes et al.[30] differ from the result of other groups in the range from $3000 \mathrm{~K}$ to $8000 \mathrm{~K}$ and show good agreement with our model above $8000 \mathrm{~K}$. The results of Abdelhakim et al.[24] show differences from $8000 \mathrm{~K}$ to 15000 K. Above $15000 \mathrm{~K}$ only results from Shayler et al. are available [25]. In this temperature range our calculations show a higher electrical conductivity. This is probably due to the consideration of higher ionized species in this work.

In Figure 10 the thermal conductivity of the $\mathrm{Cu} / \mathrm{N}_{2}$ mixture is plotted. It is noticeable that Shayler et al. calculate a similar thermal conductivity like pure nitrogen. The results of Abdelhakim et al. show a greater influence by 
the metal contamination on pure nitrogen. The thermal conductivity between $5000 \mathrm{~K}$ and $8000 \mathrm{~K}$ is lower compared with Shayler et al. and our results. The results of the presented transport model differ above $8000 \mathrm{~K}$ from the other models. In this temperature range both reference models show similar results like pure nitrogen and our results for the $\mathrm{Cu} / \mathrm{N}_{2}$ mixtures are lower. Studies with pure metal vapors show that the peaks of high thermal conductivity which result from ionization are less distinctive. Also the average thermal conductivity of metal vapor is lower than for inert gases. This should lead to lower peaks and lower average thermal conductivity for the metal/inert-gas mixture than for pure nitrogen. Probably Shayler et al. and Abdelhakim et al. underestimate the influence of iron on the thermal conductivity in this temperature range.

\subsubsection{Metal/Argon Mixtures}

The calculated electrical conductivities of binary mixtures of argon with $10 \%$ iron and $5 \%$ copper are plotted in Fig. 11. The comparison with Gonzales et al.[32] and Dunn et al. for the Fe/Ar mixture and Cressault et al.[31] and Mosthaghimi et al. for the $\mathrm{Cu} / \mathrm{Ar}$ mixture shows a good agreement over the whole temperature range.

Figure 12 shows the thermal conductivity of the argon mixtures containing $10 \%$ iron and $5 \%$ copper. The greatest influence of metal vapor is seen below $15000 \mathrm{~K}$. Above this temperature the thermal conductivities of pure argon and a metal/argon mixture are quite similar. The comparison with other research groups shows a good agreement. Only Cressault et al. apparently present different results. They report a much higher thermal conductivity above $15000 \mathrm{~K}$. 
The reason for this might be that they have computed a higher translational thermal conductivity of the electrons.

\section{Conclusion}

We predict transport properties of metal/inert-gas mixtures in the temperature range from $300 \mathrm{~K}$ to $30000 \mathrm{~K}$, which are important for arc welding processes. As metals we consider iron, copper, aluminium and calcium and as inert gases nitrogen, argon and helium.

The main findings are:

1. The analysis of the equilibrium compositions is very useful in terms of model reduction for the transport properties simulations. As we could see, based on a 43 species model for the determination of different metal/inert gas mixture compositions, only 35 species are sufficient for an excellent prediction of transport properties.

2. The model presented has been validated by comparison with well documented publications on transport coefficients in similar systems. In addition, the comparison with several metal/inert-gas models, not yet published, gives us confidence in the procedure applied for implementing higher ionized species and further support to the predictive capabilities of our transport model.

3. Addition of metal to an inert gas have a great influence on transport properties. Calcium contamination causes the greatest effect followed by aluminium, iron and copper.

4. Already a small addition of calcium, which represents metals with low ion- 
ization potential, to an iron/inert-gas mixture shows a significant influence on the electrical conductivity.

In the future the model will be implemented in $2 \mathrm{D}$ calculations to simulate gas-metal arc welding. With this model the arc welding of different steel compositions can be described. It will also be possible to optimize arc welding processes by making parametric studies e. g. finding the optimal inert gas composition.

\section{References}

[1] S. Selle, U. Riedel, Ann. N. Y. Acad. Sci. 891 (1999) 72-80.

[2] J. Prager, G. Baldea, U. Riedel, J. Warnatz, Equilibrium Composition and Electrical Conductivity of High-Temperature Air, 21st ICDERS (2007).

[3] J. Prager, U. Riedel, J. Warnatz, Proc. Combust. Inst. 31 (2007) 1129-1137.

[4] U. Maas, Mathematische Modellierung instationärer Verbrennungsprozesse unter Verwendung detaillierter Reaktionsmechanismen, $\mathrm{PhD}$ thesis, Universität Heidelberg, Heidelberg, 1988.

[5] O. Deutschmann, S. Tischer, C. Correa, D. Chatterjee, S. Kleditzsch, V. M. Janardhanan, DETCHEM - Usermanual, Karlsruhe, 2004.

[6] B. J. McBride, M. J. Zehe, S. Gordon, Report TP-2002-211556, NASA, 2002.

[7] B. Cheynet, J. D. Dubois, IDT: Le Salon de l'information electronique, 1997.

[8] J. O. Hirschfelder, C. F. Curtiss, R. B. Bird, John Wiley and Sons, 1964.

[9] S. Chapman, T. G. Cowling, Cambridge University Press, 1970.

[10] T. L. Gilbert, J. Chem. Phys. 49 (1986) 2640-2642. 
[11] L. Monchick, E. A. Mason, J. Chem. Phys. 35 (1961) 1676-1697.

[12] L. Monchick, Phys. Fluids 2 (1959) 695-700.

[13] L. A. Viehland, E. A. Mason, W. F. Morrison, M. R. Flannery, Atom. Data Nucl. Tables 16 (1975) 495-514.

[14] K. T. Tang, Phys. Rev. 177 (1969) 108-114.

[15] R. L. Liboff, Phys. Fluids 2 (1959) 40-46.

[16] E. A. Mason, R. J. Munn, F. J. Smith, Phys. Fluids 10 (1967) 1827-1832.

[17] E. A. Mason, E. W. McDaniel, Transport Properties of Ions in Gases, John Wiley and Sons, New York, 1988.

[18] R. S. Devoto, Phys. Fluids 3 (1966) 1230-1240.

[19] A. B. Murphy, Phys. Rev. E 48 (1993) 3594-3603.

[20] A. Ern, V. Giovangigli, Multicomponent Transport Algorithms, Springer Verlag, Berlin, 1994.

[21] J. O. Hirschfelder, Sixth Symposium (International) on Combustion, The Combustion Institute, 1978.

[22] J. Warnatz, In Numerical Methods in Laminar Flames, Vieweg Verlag, Braunschweig, 1982, 87-111.

[23] R. S. Devoto, Phys. Fluids 10 (1967) 2105-2112.

[24] H. Abdelhakim, J. P. Dinguirard, S. Vacquie, J. Phys. D: Appl. Phys. 13 (1980) $1427-1438$.

[25] P. J. Shayler, M. T. C. Fang, J. Phys. D: Appl. Phys. 10 (1977) 1659-1669.

[26] J. Mostaghimi-Tehrani, E. Pfender, Plasma Chem and Plasma Process 4 (1984) 129-139. 
[27] A. Aubreton, M. F. Elchinger, J. Phys. D: Appl. Phys. 36 (2003) 1798-1805.

[28] B. Chervy, O. Dupont, A. Gleizes, P. Krenek, J. Phys. D: Appl. Phys. 28 (1985) 2060-2066.

[29] H. Wilhelmi, W. Lyhs, E. Pfender, Plasma Chem and Plasma Process 4 (1984) 315-323.

[30] A. Gleizes, A. M. A. Amry, A. M. Rahal, S. Vacquié, J. Appl. Phys. 67 (1989) 663-670.

[31] Y. Cressault, A. Gleizes, J. Phys. D: Appl. Phys. 37 (2004) 560-572.

[32] J. J. Gonzales, A. Gleizes, J. Phys. D: Appl. Phys. 74 (1993) 3065-3070.

[33] G. J. Dunn, T. W. Eagar, Metall. Trans. A 17A (1985) 1865-1871. 


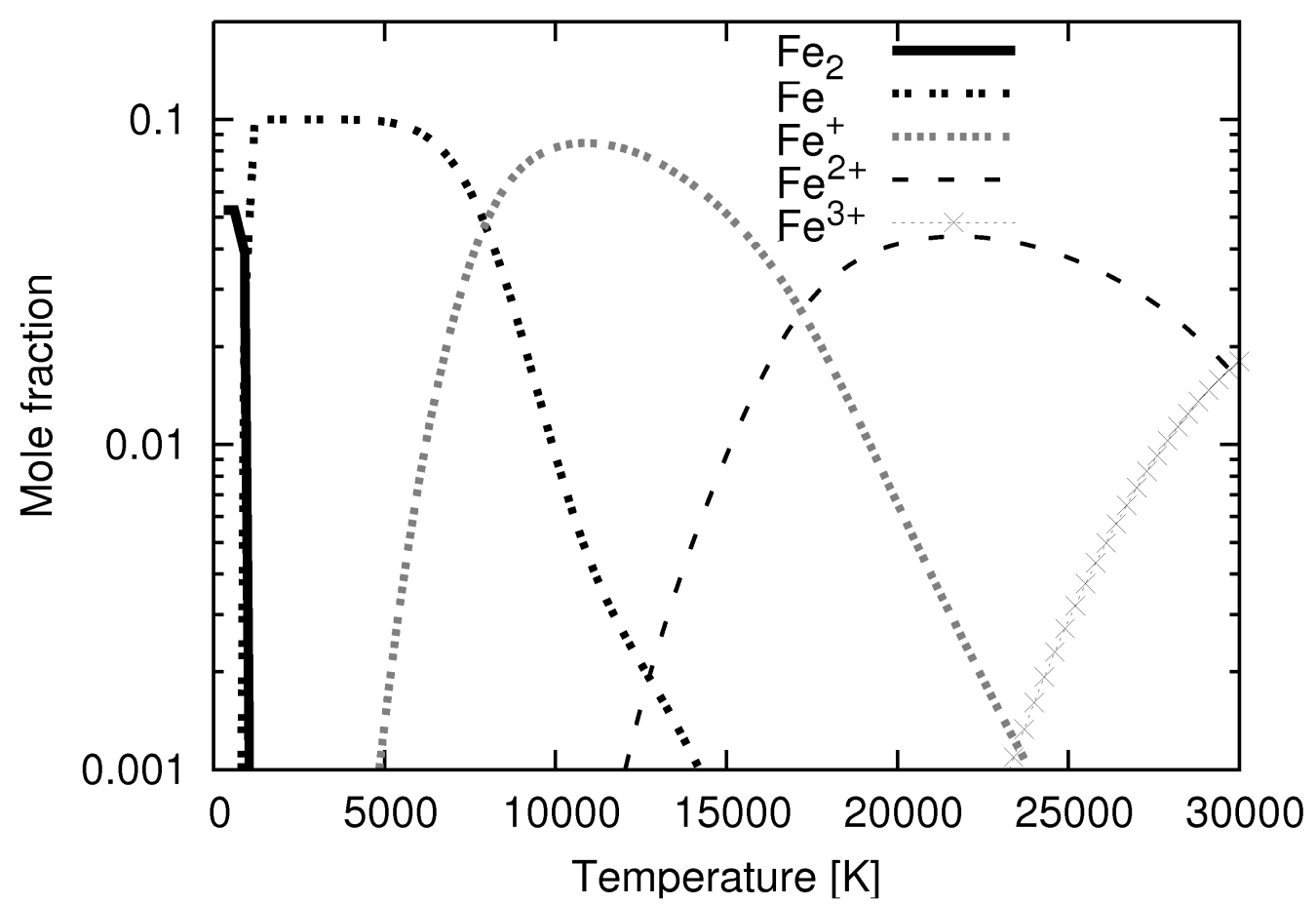

Fig. 1. Concentration profiles for relevant iron species (10\% Fe / $90 \%$ Ar mixture at 1 bar). 


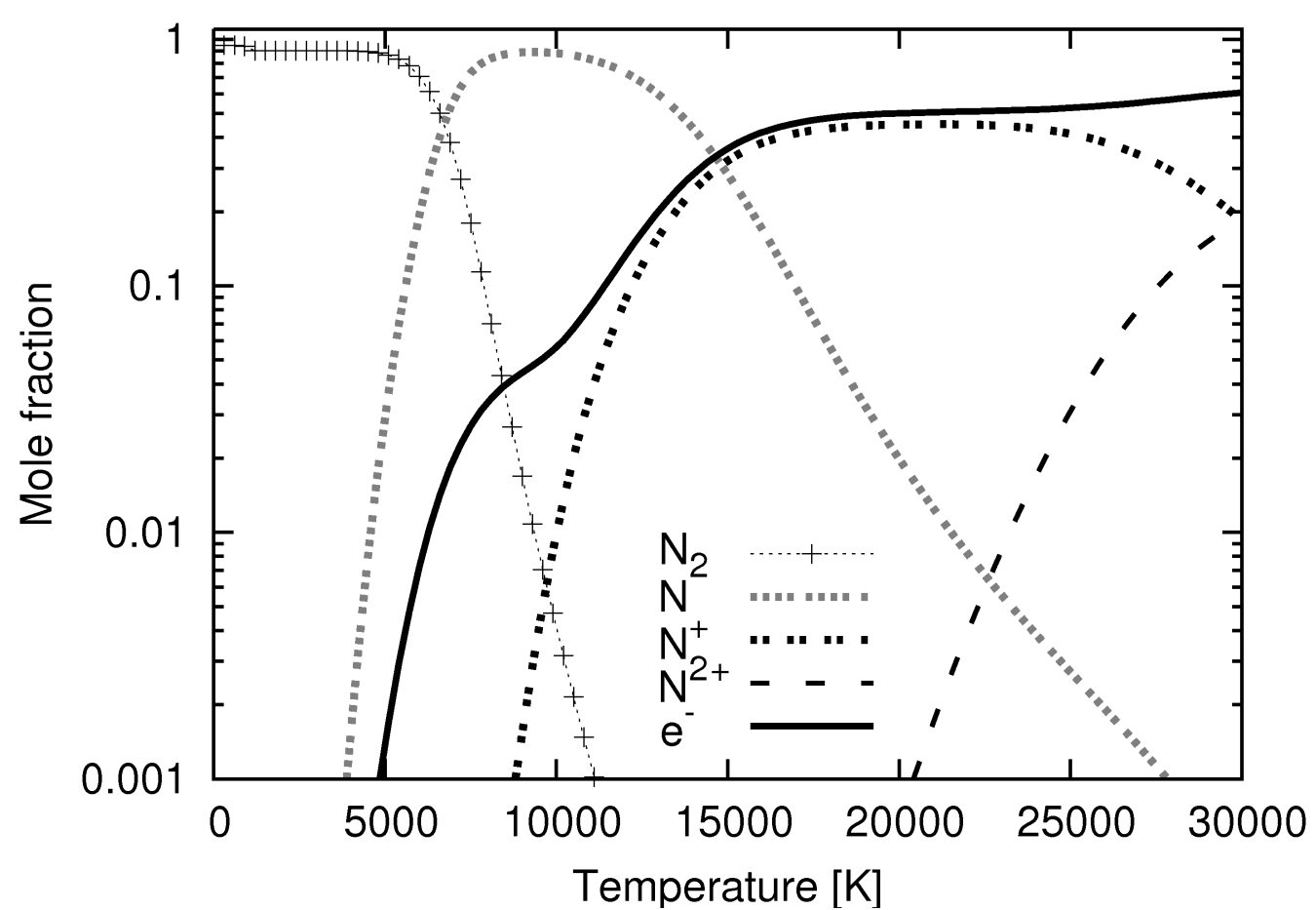

Fig. 2. Concentration profiles for relevant nitrogen species $\left(10 \% \mathrm{Fe} / 90 \% \mathrm{~N}_{2}\right.$ mixture at 1 bar). 


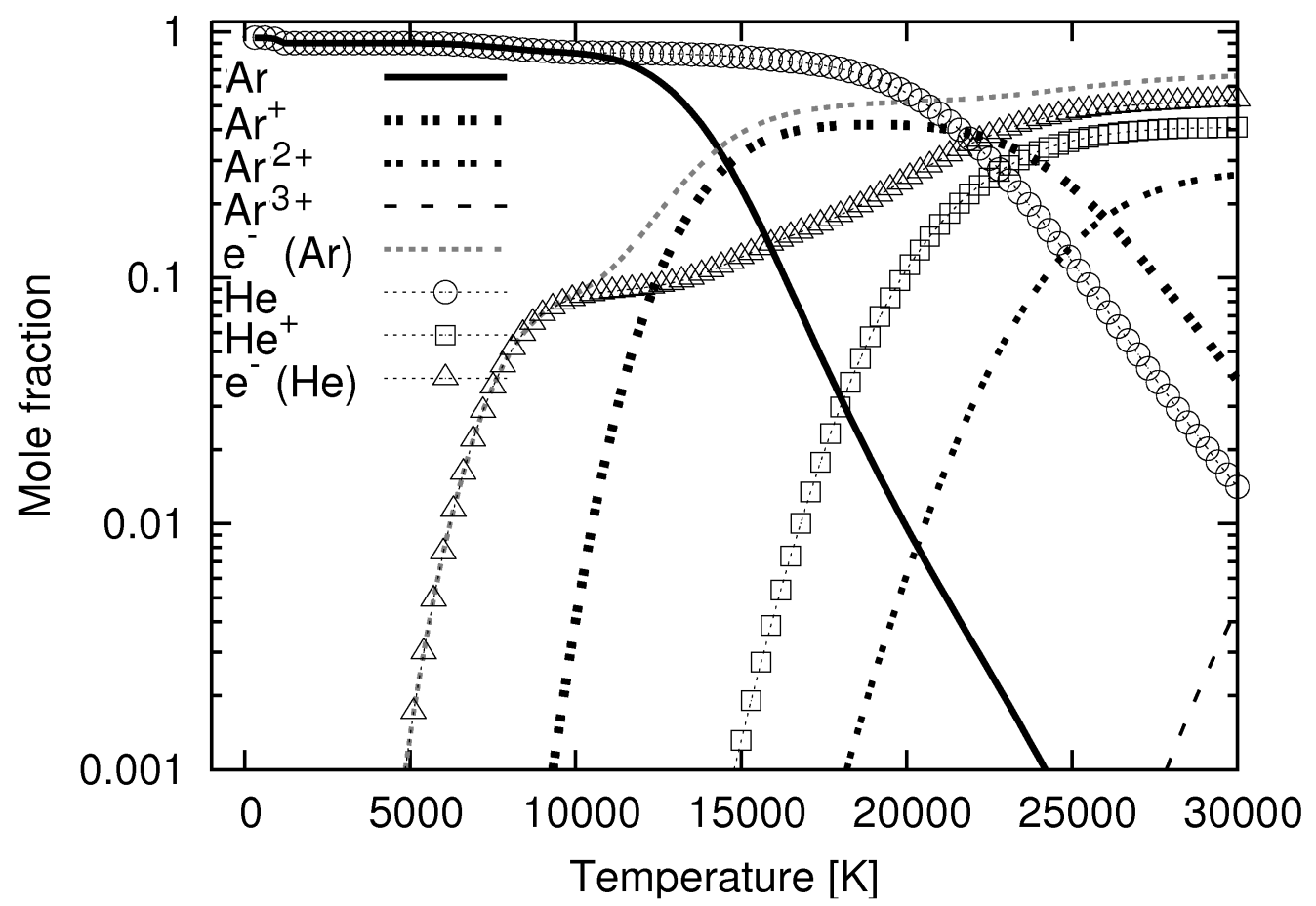

Fig. 3. Concentration profiles for relevant argon and helium species (10 \% Fe / 90 $\%$ inert-gas mixtures at 1 bar). 


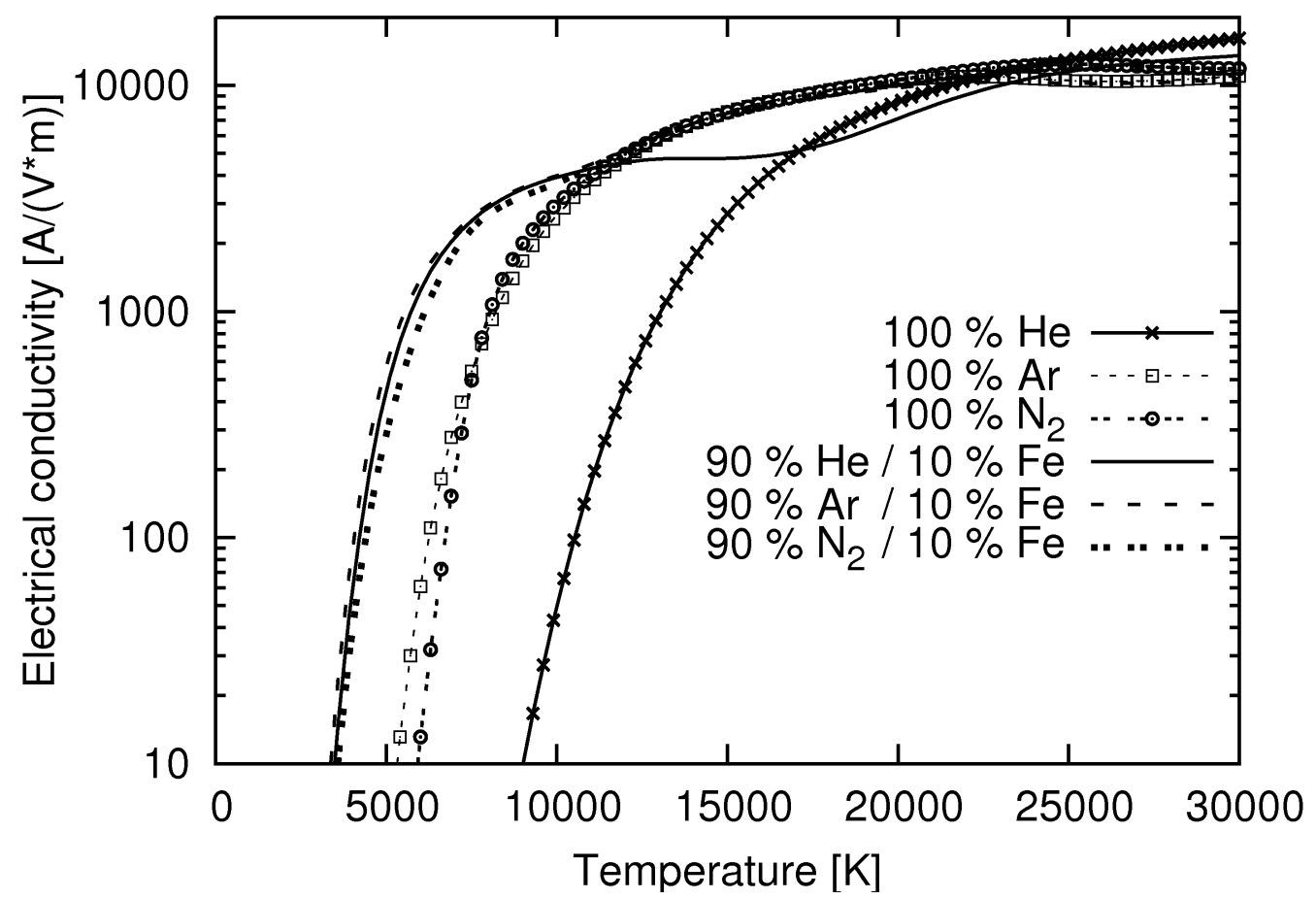

Fig. 4. Electrical conductivity of iron/inert-gas mixtures at 1 bar. 


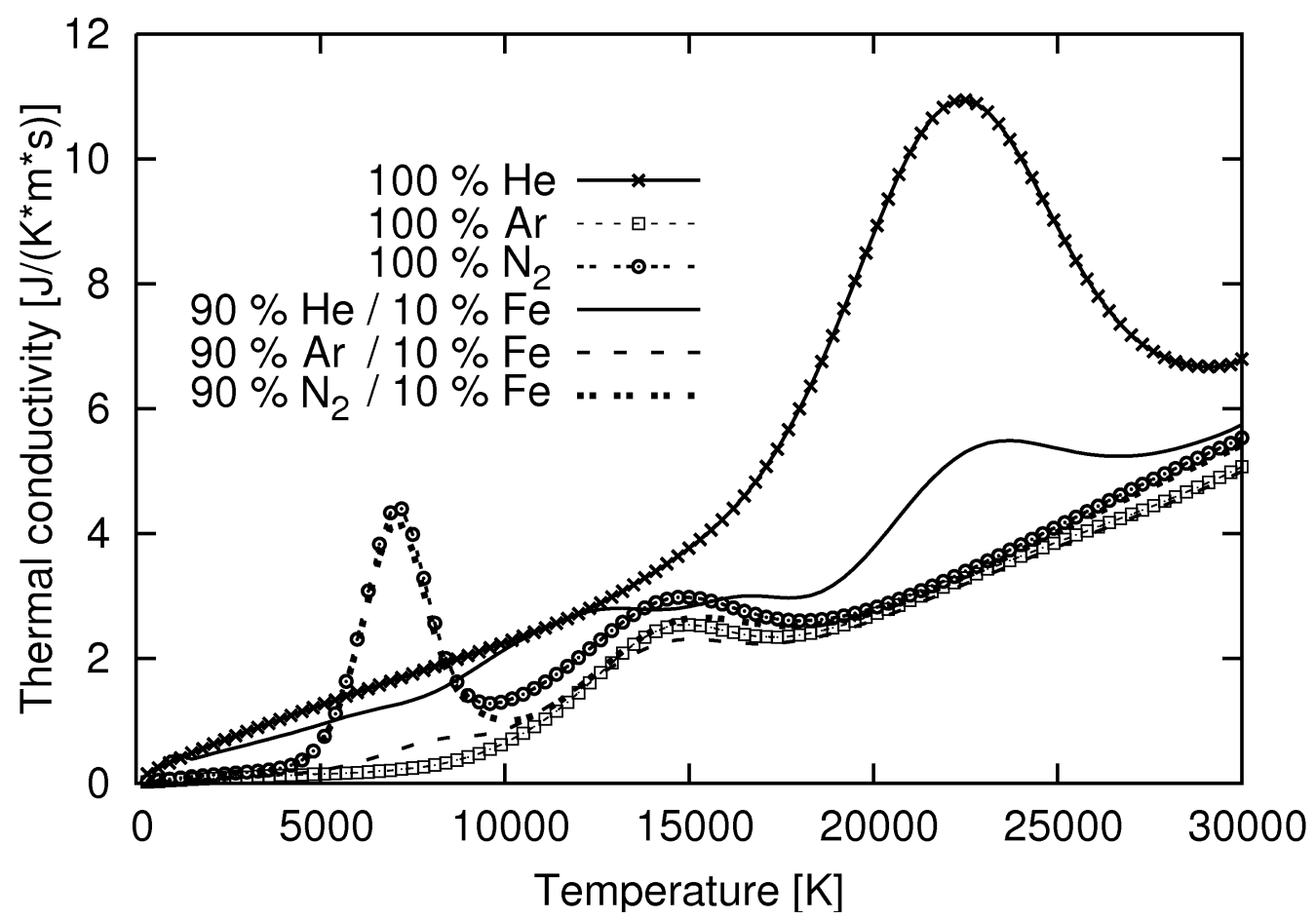

Fig. 5. Thermal conductivity of iron/inert-gas mixtures at 1 bar). 


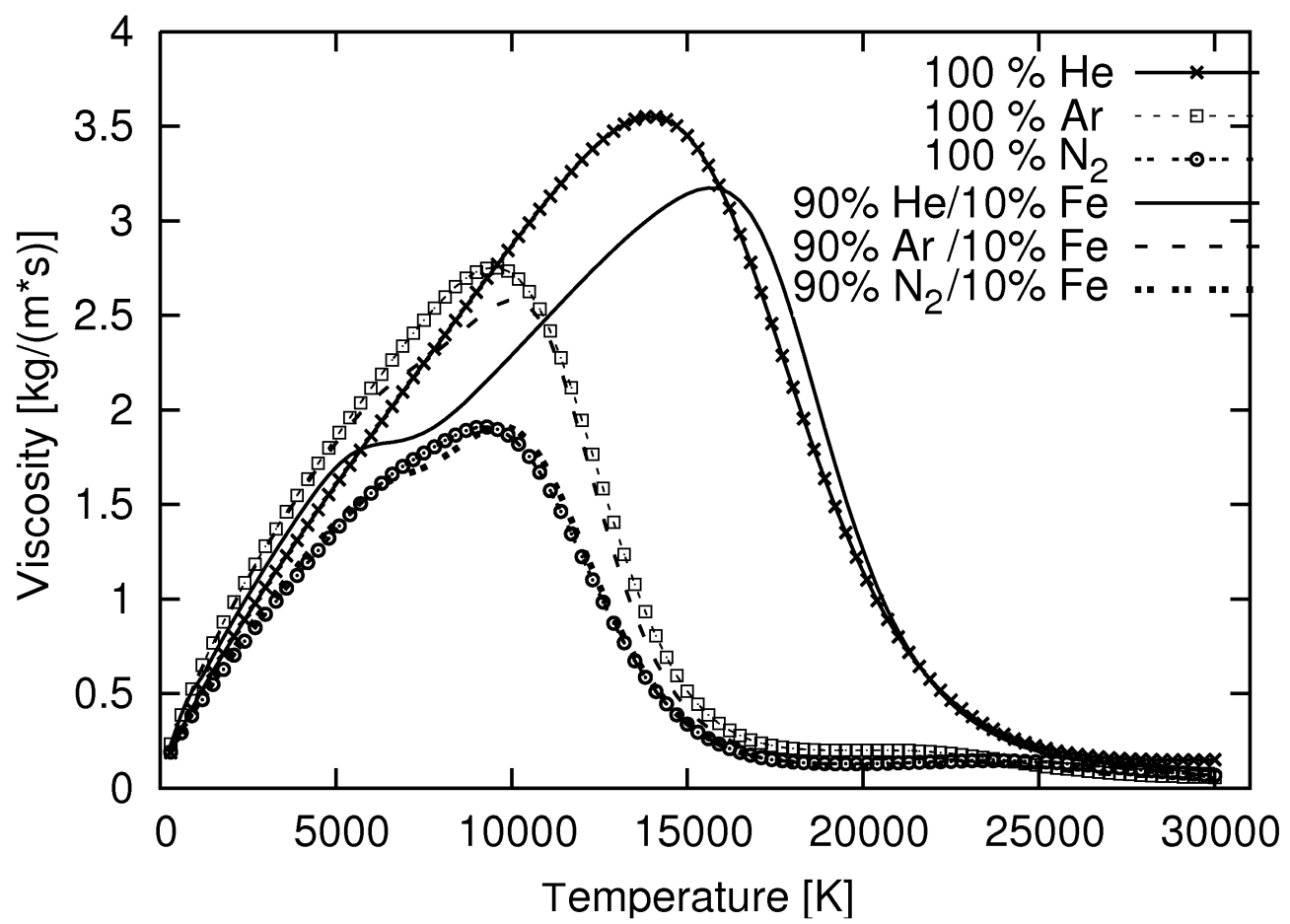

Fig. 6. Viscosity of iron/inert-gas mixtures at 1 bar. 


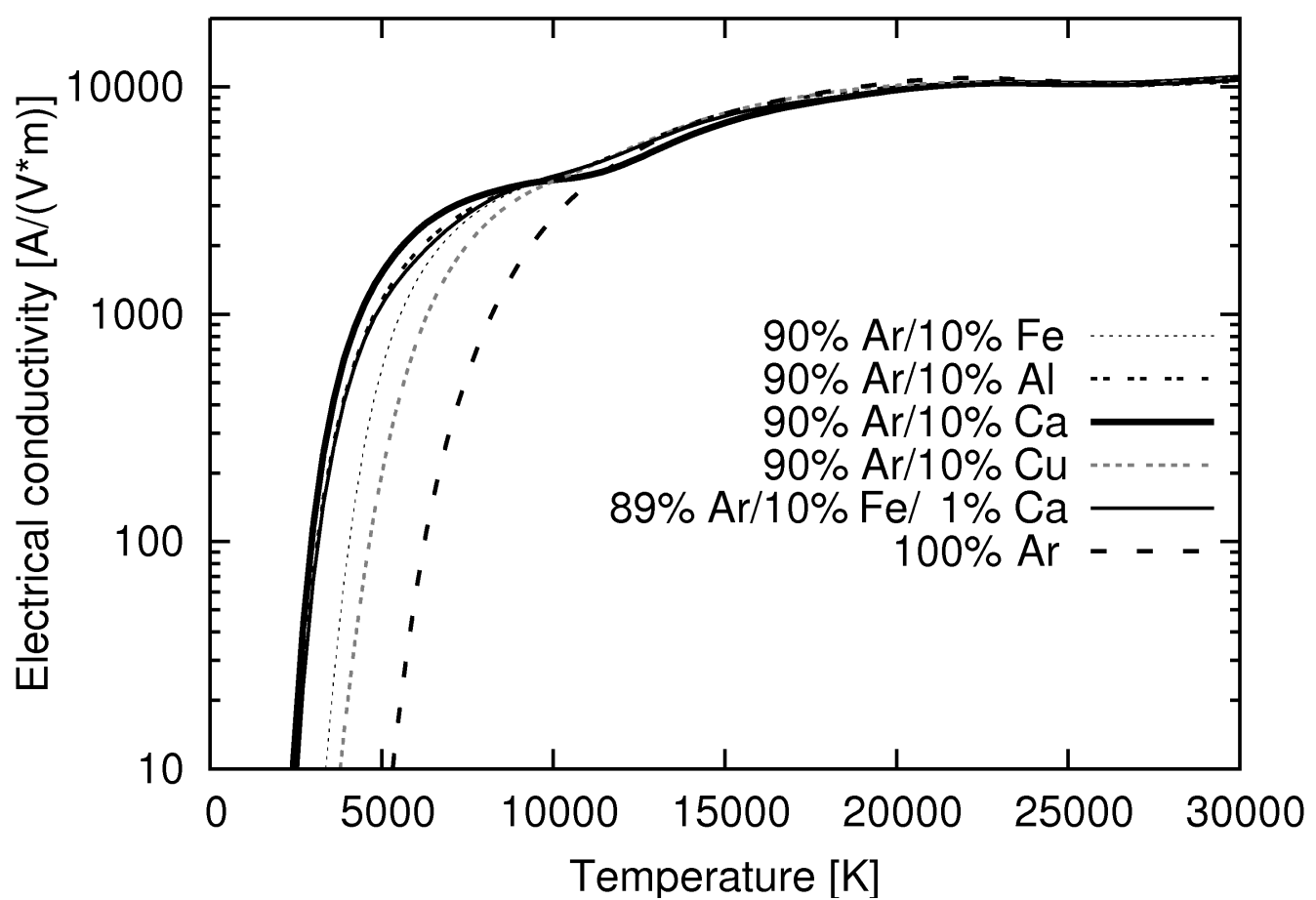

Fig. 7. Electrical conductivity of iron/inert-gas mixtures (10 \% Fe / $90 \%$ inert-gas mixtures at 1 bar). 


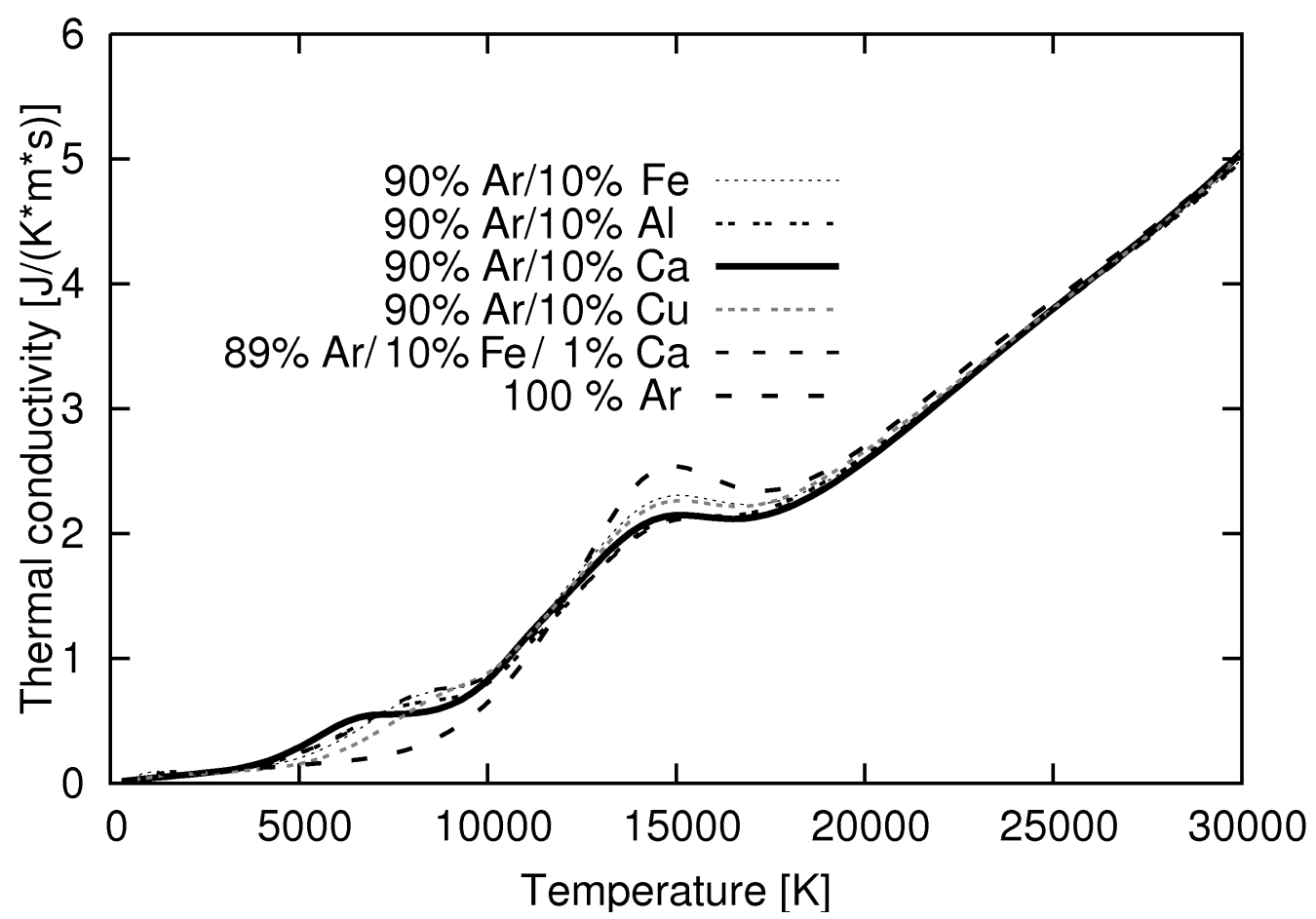

Fig. 8. Thermal conductivity of metal/inert-gas mixtures (10\% metal / $90 \%$ inert-gas mixtures at 1 bar). 


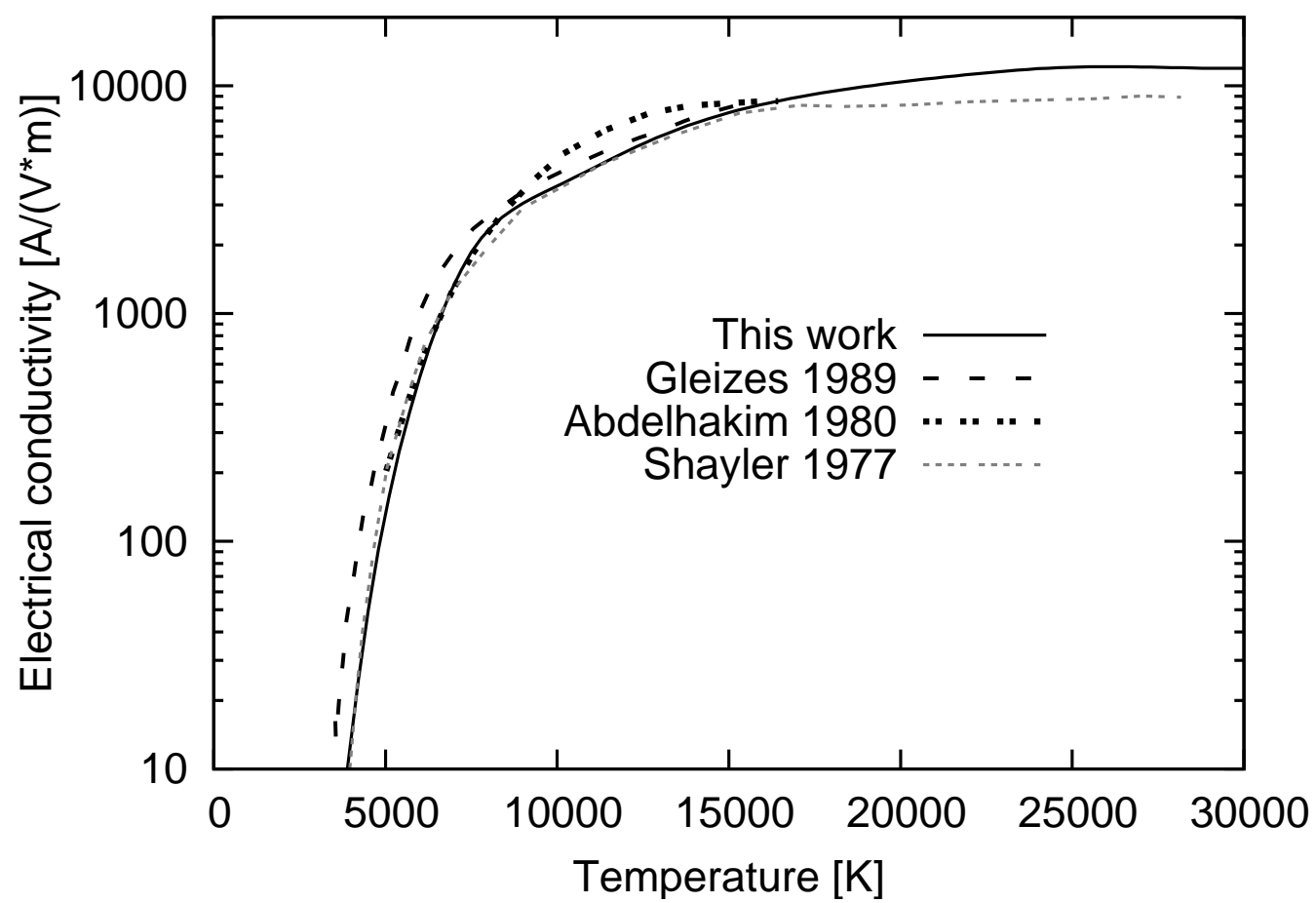

Fig. 9. Electrical conductivity of a $\mathrm{Cu} / \mathrm{N}_{2}$ mixture $\left(10 \% \mathrm{Cu} / 90 \% \mathrm{~N}_{2}\right.$ mixture at 1 bar). 


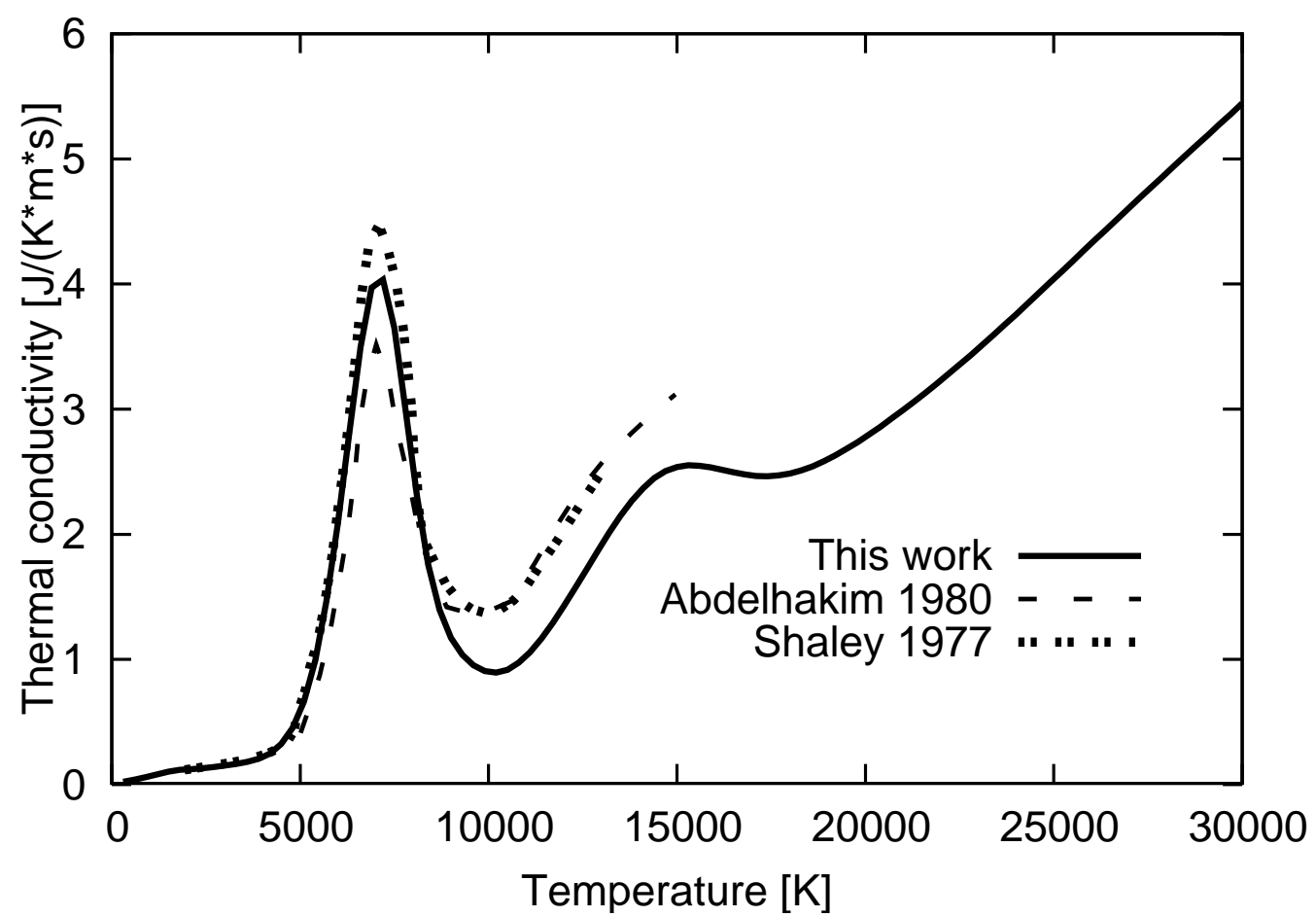

Fig. 10. Thermal conductivity of a $\mathrm{Cu} / \mathrm{N}_{2}$ mixture $\left(10 \% \mathrm{Cu} / 90 \% \mathrm{~N}_{2}\right.$ mixture at 1 bar). 


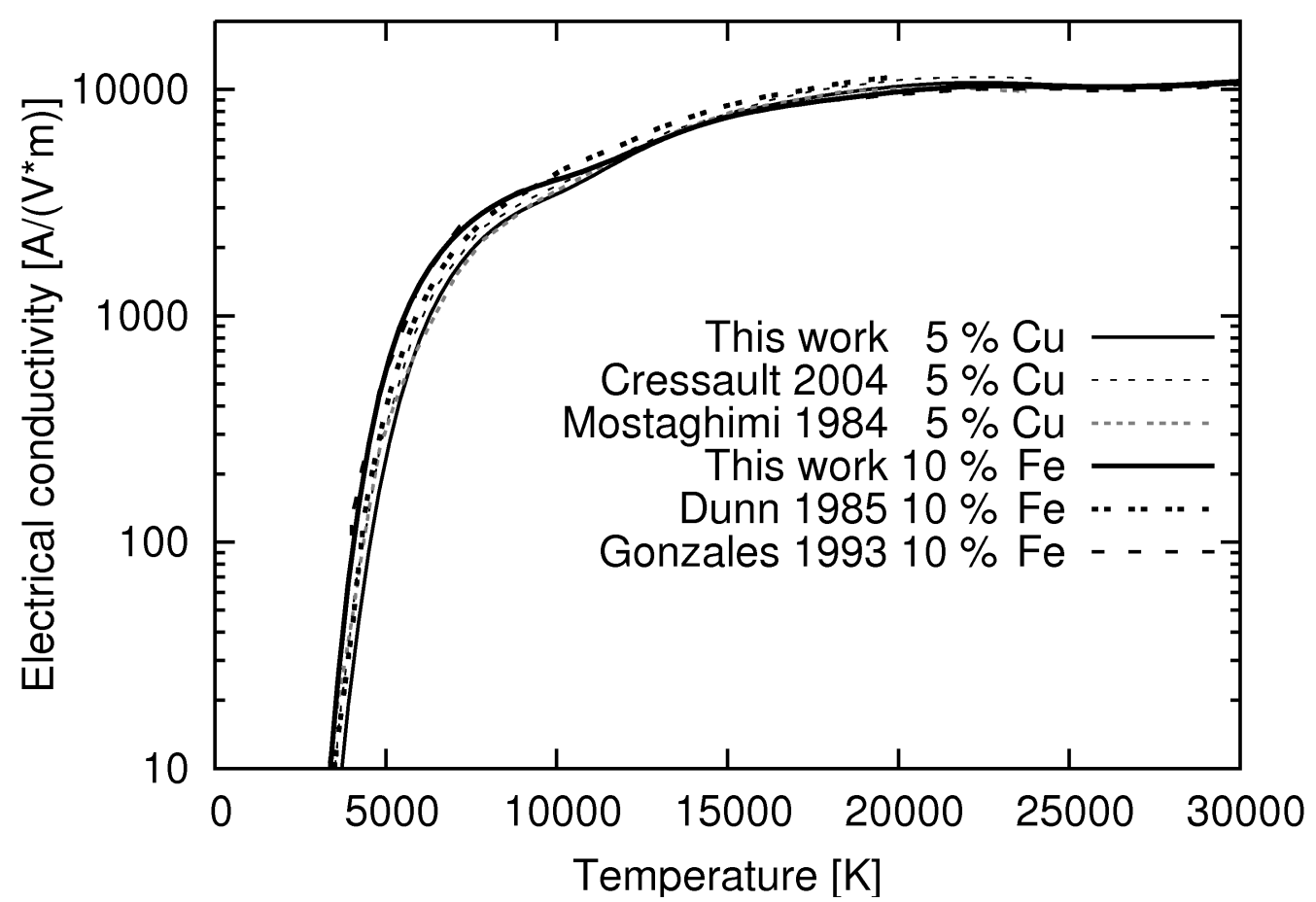

Fig. 11. Electrical conductivity of $\mathrm{Cu} /-$ and $\mathrm{Fe} / \mathrm{Ar}$ mixtures 1 bar. 


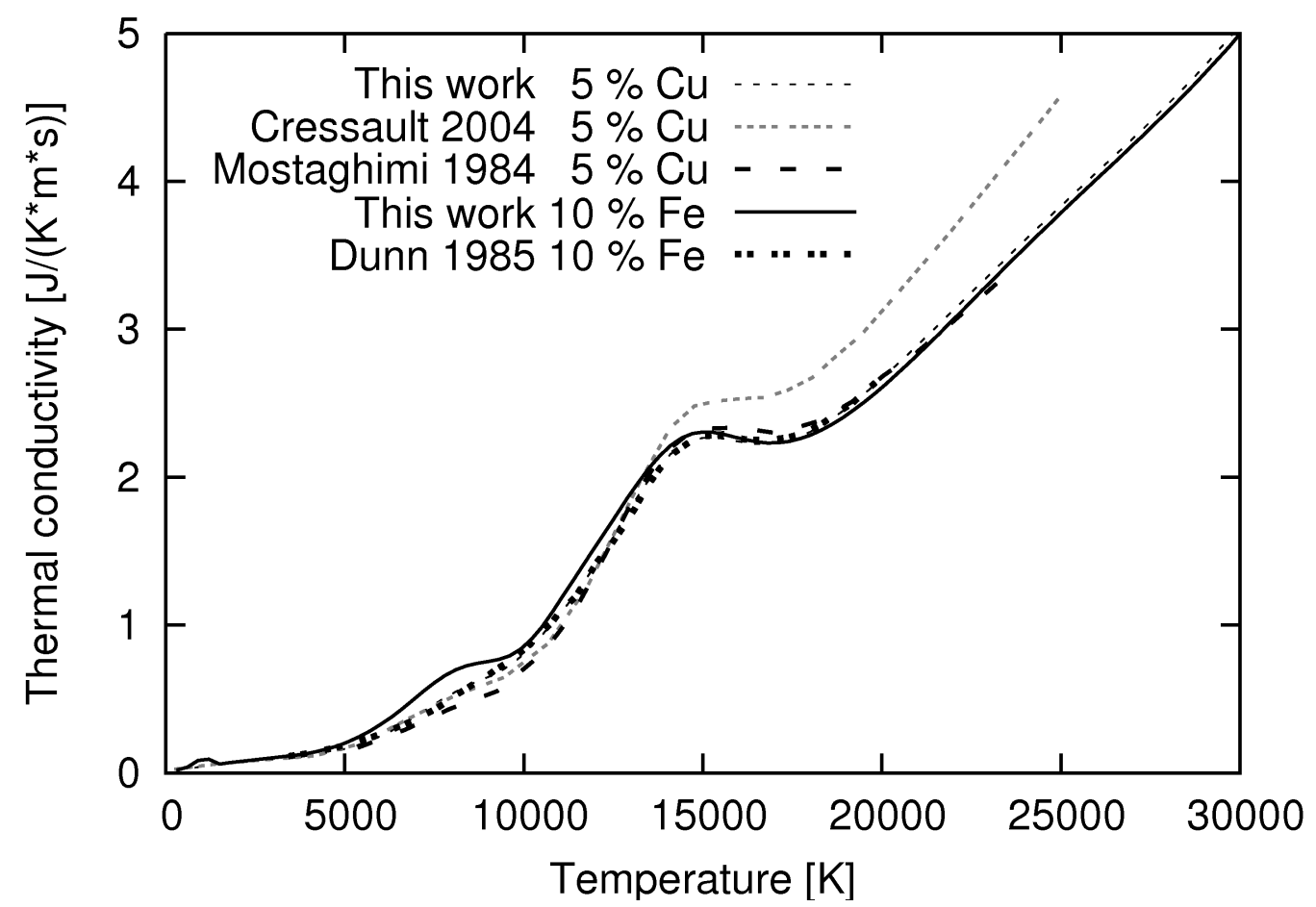

Fig. 12. Thermal conductivity of a $\mathrm{Cu} /-$ and $\mathrm{Fe} / \mathrm{Ar}$ gas mixture at 1 bar. 


\section{Figure captions}

Fig. 1: Concentration profiles for relevant iron species $(10 \% \mathrm{Fe} / 90 \% \mathrm{Ar}$ mixture at 1 bar).

Fig. 2: Concentration profiles for relevant argon and helium species (10 \% Fe / $90 \%$ inert-gas mixtures at 1 bar).

Fig. 3: Concentration profiles for relevant nitrogen species $(10 \% \mathrm{Fe} / 90 \%$ $\mathrm{N}_{2}$ mixture at 1 bar).

Fig. 4: Electrical conductivity of iron/inert-gas mixtures at 1 bar.

Fig. 5: Thermal conductivity of iron/inert-gas mixtures at 1 bar).

Fig. 6: Viscosity of iron/inert-gas mixtures at 1 bar.

Fig. 7: Electrical conductivity of iron/inert-gas mixtures (10 \% Fe / $90 \%$ inert-gas mixtures at 1 bar).

Fig. 8: Thermal conductivity of metal/inert-gas mixtures (10\% metal / 90 $\%$ inert-gas mixtures at 1 bar).

Fig. 9: Electrical conductivity of a $\mathrm{Cu} / \mathrm{N}_{2}$ mixture $\left(10 \% \mathrm{Cu} / 90 \% \mathrm{~N}_{2}\right.$ mixture at 1 bar).

Fig. 10: Thermal conductivity of a $\mathrm{Cu} / \mathrm{N}_{2}$ gas mixture $\left(10 \% \mathrm{Cu} / 90 \% \mathrm{~N}_{2}\right.$ mixture at 1 bar).

Fig. 11: Electrical conductivity of $\mathrm{Cu} /-$ and $\mathrm{Fe} / \mathrm{Ar}$ mixtures at 1 bar.

Fig. 12: Thermal conductivity of a $\mathrm{Cu} /-$ and $\mathrm{Fe} / \mathrm{Ar}$ gas mixture at 1 bar. 